\section{AURORA PHASE 3 STUDY DEMONSTRATES VOCLOSPORIN STATISTICAL SUPERIORITY OVER STANDARD OF CARE IN LUPUS NEPHRITIS (LN)}

${ }^{1}$ YK Onno Teng, ${ }^{2}$ Samir V Parikh, ${ }^{3}$ Amit Saxena, ${ }^{4}$ Neil Solomons, ${ }^{4}$ Robert B Huizinga. ${ }^{1}$ Dept. of Nephrology, Leiden University Medical Centre, Leiden, The Netherlands; ${ }^{2}$ Division of Nephrology, The Ohio State University Wexner Medical Center Columbus, OH; ${ }^{3} \mathrm{NYU}$ School of Medicine, New York, NY, USA; ${ }^{4}$ Clinical, Aurinia Pharmaceuticals, Victoria, Canada

\subsection{6/lupus-2020-eurolupus.24}

Background Voclosporin (VCS) is a novel high potency calcineurin inhibitor (CNI) with a favorable metabolic profile and a consistent predictable dose response potentially eliminating the need for therapeutic drug monitoring. The recently completed Phase 3 AURORA study builds on the favorable efficacy seen in the Phase 2 AURA-LV study in patients with active LN. The AURORA study was conducted in active LN patients to evaluate the efficacy and safety of VCS vs placebo in combination with mycophenolate mofetil (MMF, $2 \mathrm{~g} /$ day) and rapidly tapered oral steroids.

Methods AURORA is a global, randomized double-blind, placebo-controlled Phase 3 study with active LN. Key inclusion criteria include biopsy proven LN (Class III, IV, V) and proteinuria of $\geq 1.5 \mathrm{mg} / \mathrm{mg}$ or $\geq 2 \mathrm{mg} / \mathrm{mg}$ for Class $\mathrm{V}$ patients. The primary endpoint was renal response (RR) at 52 weeks defined as UPCR of $\leq 0.5 \mathrm{mg} / \mathrm{mg}$, eGFR $\geq 60 \mathrm{~mL} / \mathrm{min}$ or no confirmed decrease from baseline in eGFR of $>20 \%$, presence of sustained, low-dose steroids and no administration of rescue medication.

Results AURORA enrolled 357 adult LN patients. The RR rate was $40.8 \%$ for voclosporin versus $22.5 \%$ for control (OR: 2.65; 95\% CI: 1.64, 4.27; p<0.001). Also, a significantly higher proportion of LN patients achieved pre-specified hierarchical secondary endpoints for voclosporin including: RR at 24 weeks, partial renal response (PRR) at 24 and 52 weeks, time to achieve UPCR $\leq 0.5 \mathrm{mg} / \mathrm{mg}$, and time to $50 \%$ reduction in UPCR (table 1). The efficacy benefit of VCS on RR was seen across prespecified biopsy subgroups: for pure Class V LN (OR: 2.74; 95\% CI: 0.78, 9.68), and for Class III/IV alone or in combination with Class V patients (OR: 2.63; 95\% CI: 1.57, 4.41). Furthermore, all pre-specified subgroup analyses (age, sex, race, region, and prior MMF use) also favored VCS.

The overall incidence of SAEs was similar in both groups (VCS 20.8\% and control 21.3\%), with infections most commonly reported (VCS 10.1\% and control 11.2\%). Overall mortality in the trial was low, with six deaths observed; one in the voclosporin arm and five in the control group. Additionally, at Week 52 the VCS arm showed no significant decrease in eGFR or increase in BP, lipids or glucose.

Conclusion The addition of VCS to MMF and low-dose steroids demonstrated superior efficacy to standard of care in active LN patients. The 104-week double-blind AURORA continuation study will provide longer term safety and efficacy data.

\section{REMISSION AND LLDAS AS A TARGET FOR PREGNANCY PLANNING IN SLE?}

'Dina Zucchi, 'Chiara Tani, ${ }^{2}$ Francesca Monacci, 'Elena Elefante, 'Linda Carli, 'Chiara Stagnaro, ${ }^{1}$ Alice Parma, ${ }^{3}$ May Choi, ${ }^{1}$ Francesco Ferro, ${ }^{1}$ Viola Signorini, ${ }^{1}$ Sabrina Gori, ${ }^{2}$ Francesca Strigini, ${ }^{1}$ Marta Mosca. ${ }^{1}$ Rheumatology Unit, Dept. of Clinical and Experimental Medicine, University of Pisa; ${ }^{2}$ Division of Gynecology and Obstetrics, Dept. of Clinical and Experimental Medicine, University of Pisa, Italy; ${ }^{3}$ University of Calgary, Cumming School of Medicine, Calgary, Canada

\subsection{6/lupus-2020-eurolupus.25}

Background The attainment of remission and low disease activity (LLDAS) in SLE is associated with better outcomes. This study was aimed to test whether disease remission and LLDAS at the beginning of pregnancy are associated with better pregnancy outcomes.

Methods A total of 85 pregnancies in 61 SLE patients prospectively followed at a single center were considered; 10 pregnancies ended in spontaneous abortion during the first trimester and were excluded from the analysis. Definitions of remission (according to DORIS criteria) and LLDAS were applied at the first pregnancy visit (7-8 weeks of gestational age). Disease flare was defined according to the SELENA-SLEDAI flare index. Obstetric complications included preterm prelabour rupture of membranes, preeclampsia, preterm delivery, SGA infant, IUGR, intrauterine fetal death (IUFD) and gestational diabetes.

Results Characteristics of the cohort are detailed in table 1. In twenty-one cases (28\%) we observed flare during pregnancy or puerperium. Two severe flares and 19 mild-moderate flares were recorded. Obstetric complications were observed in 31 cases (41\%). The risk of disease flare during pregnancy was significantly lower in patients in LLDAS at the beginning of

Abstract 011 Table 1 Primary and hierarchal secondary outcomes

\begin{tabular}{|c|c|c|c|c|}
\hline Hierarchical Sec & Primary and Hierarchal Secondary Outcomes & Result & $\begin{array}{l}\text { Odds Ratio } \\
{[95 \% \mathrm{Cl}]}\end{array}$ & p-value \\
\hline \multirow[t]{2}{*}{ Primary Endpoint } & Renal Response at 52 weeks & Voclosporin $40.8 \%$ & $2.65[1.64,4.27]$ & $p<0.001$ \\
\hline & & Control $22.5 \%$ & & \\
\hline \multirow[t]{10}{*}{ Secondary Endpoints } & Renal Response at 24 weeks & Voclosporin $32.4 \%$ & $2.23[1.34,3.72]$ & $p=0.002$ \\
\hline & & Control $19.7 \%$ & & \\
\hline & Partial Renal Response at 24 weeks & Voclosporin $70.4 \%$ & $2.43[1.56,3.79]$ & $\mathrm{p}<0.001$ \\
\hline & & Control $50.0 \%$ & & \\
\hline & Partial Renal Response at 52 weeks & Voclosporin $69.8 \%$ & $2.26[1.45,3.51]$ & $\mathrm{p}<0.001$ \\
\hline & & Control $51.7 \%$ & & \\
\hline & Time to UPCR $\leq 0.5 \mathrm{mg} / \mathrm{mg}$ & Voclosporin faster than Control & $2.02[1.51,2.70]$ & $\mathrm{p}<0.001$ \\
\hline & & & Hazard Ratio & \\
\hline & Time to $50 \%$ reduction in UPCR & Voclosporin faster than Control & $2.05[1.62,2.60]$ & $\mathrm{p}<0.001$ \\
\hline & & & Hazard Ratio & \\
\hline
\end{tabular}

